\title{
ASPECTOS NEUROQUIRÚRGICOS DE LAS HERIDAS ORBITOCRANEALES
}

\author{
H. VERBIEST *
}

Nos proponemos describir algunos aspectos de las heridas orbitocraneales, tal como éstas se presentan en tiempo de paz. La segunda guerra mundial nos ha reportado enorme experiencia en lo referente a estas heridas, y importantes comunicaciones han sido publicadas (ver, entre otros, Webster y Gurdjian, Webster, Schneider y Lofström, Steward y Botterell, Calvert, Peiper, Tönnis). Sobre la base de estas publicaciones el tratamiento de las heridas orbitocraneales ha quedado establecido con arreglo a pautas bien circunscritas y determinadas.

Por lo que se refiere a dicho tratamiento, se pueden distinguir cuatro grupos diferentes de lesiones: 1) Heridas del globo ocular y de otras importantes zonas vecinas del contenido orbitario (músculos, nervios, etc.); 2) Heridas de los senos paranasales; 3) Heridas del contenido intracraneal; 4) Heridas de otras zonas de la cara.

$\mathrm{Si}$ consideramos estos diferentes tipos de lesiones podemos comprender que un buen tratamiento es sólamente posible en Centros donde cabe un trabajo de colaboración entre el oftalmólogo, el otorrinolaringólogo, el neurocirujano y el cirujano plástico. Estos especialistas no sólamente trabajan conjuntamente, sino que a veces están obligados a traspasar su propio terreno, si las circunstancias lo exigen.

Las heridas orbitocraneales de guerra difieren de aquéllas en tienıpo de paz por varias causas: 1) En las heridas de guerra existe frecuentemente pérdida del globo ocular por ruptura o tocal destrucción, a consecuencia de proyectiles o explosivos. En las heridas de paz predominan las lesiones por puñetazos, violencias, choques, etc., con objetos cuyo tamaño es mayor que el de la órbita, parándose el golpe contra los bordes de la misma. El globo ocular, que tiene una gran posibilidad de desplazamiento, escapa así frecuentemente al trauma reduciéndose la lesión, en caso de una eventual herida, la mayoría de las veces a una simple hemorragia intraocular. Las parálisis de los músculos oculares o la ceguera, en las heridas orbitarias de guerra, están lo más a menudo producidas por destrucción directa de los nervios por el proyectil, en tanto que en las heridas de paz son debidas generalmente a lesiones traumáticas de las zonas por donde estos nervios penetran en la órbita, es decir, a nivel de la fisura orbital

* Lector de Neurocirugía de la Universidad de Utrecht. 
superior y del agujero óptico. 2) En las heridas de guerra penetran cascos de metralla o proyectiles con la suciedad que los acompaña. La especial localización del proyectil y el camino por el que el cuerpo extraño penetra en el cráreo, forman problemas especiales que no se presentan en los accidentes de la circulación y del trabajo.

En tiempo de paz las más frecuentes complicaciones de las heridas órbitocraneales que requieren ser tratadas por el neurocirujano son: A) Heridas del cerebro y sus membranas; B) Comunicaciones traumáticas anormales con los senos paranasales; C) Formación de comunicaciones cráneo-orbitales anormales, de origen traumático, producidas de dentro hacia fuera; D) Determinadas deformaciones de la cavidad orbitaria.

En los casos A y B es necesario lo más a menudo un tratamiento inmediato. Por lo que se refiere a estas heridas la literatura les ha prestado la mayor atención y las reglas de su tratamiento están internacionalmente estandardizadas. Para tratar sobre este tema nos ha parecido útil hablar en un párrafo aparte de las heridas intracraneales transorbitarias penetrantes. En los citados casos C y D los síntomas se manifiestan más tardiamente, no siendo necesario operar inmediatamente.

A. Heridas cerebrales - El tratamiento de las heridas del cerebro y de la duramadre, como complicación de las heridas orbitarias, está fundamentado en los mismos principios que su tratamiento en cualquier otra parte del cráneo. Debe procederse a la extirpación de los coágulos, de los cuerpos extraños y del tejido cerebral lesionado y desvitalizado. Después de una cuidadosa hemostasia debe cerrarse completamente la dura, haciendo, en caso necesario, una transplantación de fascia.

Las normas del traiamiento operatorio de las heridas intracraneales están determinadas detalladamente por las siguientes circunstancias: 1) La naayoría de las veces se llega a la herida cerebral por medio de una trepanación hecha a nivel del centro de la herida. En las heridas de la base del lóbulo frontal, que van unidas lo más a menudo a una fratura comu. nicante o comunitiva del techo de la órbita, debe tratarse la herida cerebral por un camino transorbitario, lo que también constituye un buen método en las heridas, más extensas, de guerra. Sobre todo se ha demostrado en la segunda guerra mundial que una trepanación sobre el lóbulo frontal permite, la mayoría de las veces, una mejor vía de entrada, por. que, después de abrir la dura y separar hacia arriba el lóbulo frontal, se puede ver y tratar mucho mejor la herida cerebral y la rotura de: la duramadre. Si se levaita después de esto con una espátula la dura de la base de la fosa anterior se puede ver también la herida del techo de la órbita y la comunicación producida traumáticamente entre el seno y la cavidad craneal. 2) El cierre de la dura es en estos casos aún de mayor importancia que en las heridas en otra parte del cráneo. En el tipo de lesión citado últimamente debe cerrarse el defecto de piel encima de la dura, incluso, si es necesario, por medio de rotación de un colgajo cutáneo. En las heridas órbitocraneales la dura forma frecuentemente la única barrera 
contra el seno abierto, que constituye además una fuente de infección. Webster y Gurdjian emplean por eso en algunos casos en los gue no es posible cerrar bien la herida de la dura, y para prevenir una fístula de líquido, sólamente una transplantación de fascia lata con caracter temporal. Después de 15 días se quita la transplantaciónt. el espacio subaracnoideo queda cerrado por granulación y el lecho de la herida se prepara Irara una transplantación de piel. Estc se refiere sólamente a una extensa lesión de la órbita en la que la herida cerebral es visible en la superficie. Cuando existe ya una infección no es recomendable hacer una plastia de dura con fascia lata. En estos casos lo mejor es extirpar todo el techo de la órbita y abrir el espacio periorbitario. La grasa orbitaria, que se expande hacia dentro, llena el espacio muerto y cubre el defecto de dura convenientemente. En tales casos es de grán interés cuidar un buen drenaje nasal.

B. Comunicaciones de origen traumático entre los senos paranasales y el espacio intracraneal - En estos casos existe el peligro de producirse licuorrea, neumoencéfalo, meningitis y abceso cerebral. Si el seno frontal está abierto se puede extirpar fácilmente su pared posterior y toda la mucosa, con lo que el seno desaparece entrando a formar parte de la cavidad craneal. Las células etmoidales lesionadas deben extirparse, siendo necesario cuidar un buen drenaje nasal.

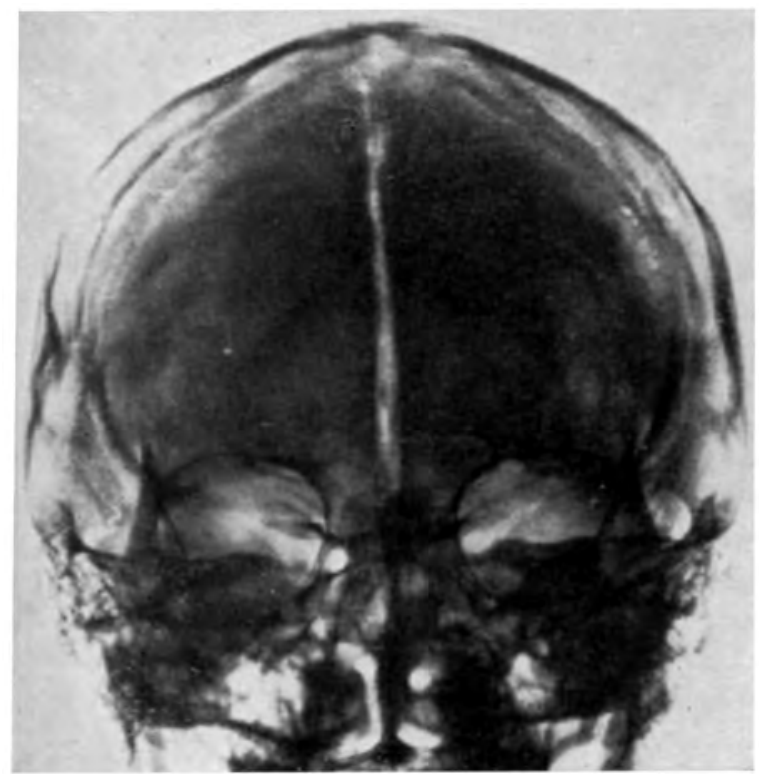

Fig. 1 - Neumoencéfalo postraumático: se observa aire a lo largo de la hoz, a consecuencia de una fractura de las células etmoidales anteriores. 
Aún en los casos en los que las radiografías de cráneo, tomadas inmediatamente después del trauma, no muestran aire en la cavidad craneal (neumoencéfalo), éste puede aparecer en días sucesivos. Sobre todo si un tnfermo presenta, algunos días después del trauma, fenómenos de. alta hipertensión intracraneal o una fuerte alteración psíquica, es útil repetir la exploración radiológica del cráneo ya que el neumoencéfalo puede ser la causa de ello. En las lesiones del seno frontal el aire se encuentra sub. dural, encima del polo frontal, y en aquellas otras en que existe una ruptura del lóbulo se presenta aire intracerebral e incluso intraventricular si la ruptura llega hasta el ventrículo. El acúmulo de aire subaracnoideo se ve muy raramente en estos casos ya que el espacio subaracnoideo por encima del polo frontal es muy pequeño. En las rupturas en los alrededoses de las cisternas basales el aire puede penetrar en el espacio subaracnoideo e incluso llegar por vía retrógrada hasta el sistema ventricular. Esto puede presentarse en las fracturas de las células etmoidales posteriores y del seno esfenoidal. En un caso de fractura de las células etmoidales an. teriores se puede ver un neumoencéfalo a lo largo de la hoz (fig. 1).

$\mathrm{Si}$ el trauma orbitocraneal requiere un tratamiento operatorio y se hace una exploración transcraneai a través de una trepanación frontal apenas aparece fístula de líquido o neumoencéfalo. Estas son complicaciones de los casos en los que no se consideró indicado el tratamiento operatorio en la primera inspección. En semejantes circunstancias es recomendable un tratamiento conservador en los primeros días. $\mathrm{Si}$ no hay señales de recuperación espontánea debe recurrirse a la intervención operatoria para cerrar la comunicación anormal. No siempre es posible visualizar una fractura en la radiografía, por lo que, en algunos casos, esta se descubre durante la operación.

La meningitis se produce, hien por la entrada directa de suciedad a consecuencia del trauma, bien por una comunicación entre los espacios de líquido cefalorraquideo y los senos paranasales. Esta comunicación puede ser la causa de meningitis recidivantes o de una meningitis rebelde. El mejor método para prevenir la meningitis lo consituye un adecuado tratamiento de la herida y la administración de antibióticos tanto en el lecho de la herida y en las vias del líquido cefalorraquideo, como intramuscular. En las heridas orbitocraneales causadas por accidentes en el trabajo y en 'a vida civil, los atceso, cerebrales constituyen una rara y deplorable complicación que lo más a menudo es la consecuencia de no haber reconocido la existencia de una comunicación con uno o más senos. Algunas veces se produce el abceso varios años después del trauma a consecuencia de una sinusitis (en uno de nuestros enfermos, con un defecto en la pared posterior del seno frontal, 14 años después del trauma).

Para ilustrar lo tratado en los apartados A y B citamos a continuación dos historias clínicas, una de herida orbitocraneal abierta y otra de herida cerrada. En el primer enfermo la intervención neuroquirúrgica, en ausencia del autor, fué practicada por uno de sus ayudantes. El autor 
mismo no hubiera procedido a hacer un cierre plástico del defecto en el tccho de la órbita con una lámina periostiocorticai, ya que existía el riesgo de una infección en un terreno ya amenazado de ella. Sin embargo esta transplantación fué seguida de éxito.

Caso 1 - C. v. d. P., muchacho de 14 años. El 23-12-1951, algunas horas después de un trauma en la cara y en el cráneo, a consecuencia de un accidente de circulación, vino a la Clínica de Neurocirugía. No perdió conciencia ni presentó amnesia retrógrada. 'Tuvo varios vơmitos. I a piel encima de la mitad derecha de la frente estaba desgarrada siendo visible el frontal firacturato. 'También la raiz ósea de la nariz estaba al descubierto. El hueso nasal derecho estaba destruído, fluyendo abundante líquido a través de la herida. Todo e] territorio lesionado estaba mu sucio por materias de la calle. El párpado derecho muy hinchado, el bulbo ocular intacto, la visión y los movimentos oculares normales. La exploración neurológica no mostró nada de particular. Fn la radiografía de cráneo (fig. 2) se observó un neumoencéfalo subdural encima del lóbulo frontal $\mathbf{y}$ aire en las cisternas basales $y$ en los ventrículos

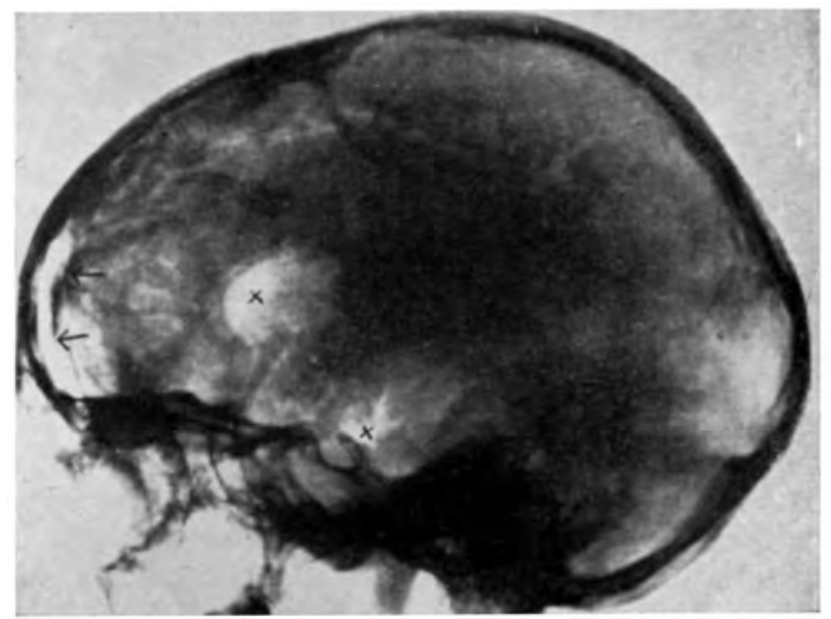

Fig. 2 - Caso 1 (C. v. d. P.). Neumoencéfalo tratmático: las filechas indican el aire subdural encima del polo frontal; las cruces se encuentran encima del aire existente en las cisternas basales $y$ en el cuerno frontal del ventrículo lateral.

Operación - Después de limpiar la herida y extirpar los tejidos desvitalizados se hizo una trepanación frontal derecha. La exploración extradural del techo de la órbita muestra que éste está destrozado y algunos trozos de hueso imprimidos en aquélla. Hay una abertura del seno frontal en su porción orbitaria. La dura está desgarrada por delante del quiasma óptico. No se observa lesión de las células etmoidales. El techo de la órbita fué repuesto y a!gunos trozos de hueso extirpados. La grasa orbitaria herniada cerró completamente la abertura de la parte orbitaria del seno frontal. Por medio de una lanuina 
periostiocortical se cerró el defecto en el techo de la órbita. La apertura dural fué suturada por vía intradural. El lóbulo frontal estaba hiperémico, pero sin ninguna lesión. Los nervios ópticos y el quiasma estaban igualmente normales. El colgajo óseo, del que se extirparon aigunos trozos fracturados, se volvió a poner en su sitio. Como estaba destruída una gran extensión de piel se hizo un colgajo de piel con cabellos, y por medio de una rotación quedó cabierta con piel toda la zona frontal traumatizada. Hil defecto de piel producido por la rotación, de localización parietooccipital, fué cubierto con piel, por medio de un 'Thierse. Durante la operación se puso penicilina, tanto intra como extraduralmente. El cirujano plástico, Dr. Honig, suturó la herida del labio superior. El otorrino, Dr. Ruding, colocó un dreno nasal en el seno frontal derecho; para la conservación de un buen drenaje.

El enfermo fué tratado postoperatoriamente con penicilina intramuscular. El curso de la herida fué bueno, y el 26-2-52 se comenzó la preparación de un colgajo de piel en forma de tubo, que por vía brazo izquierdo fué transplantado a la frente para sustituir el trozo rotado, con cabellos.

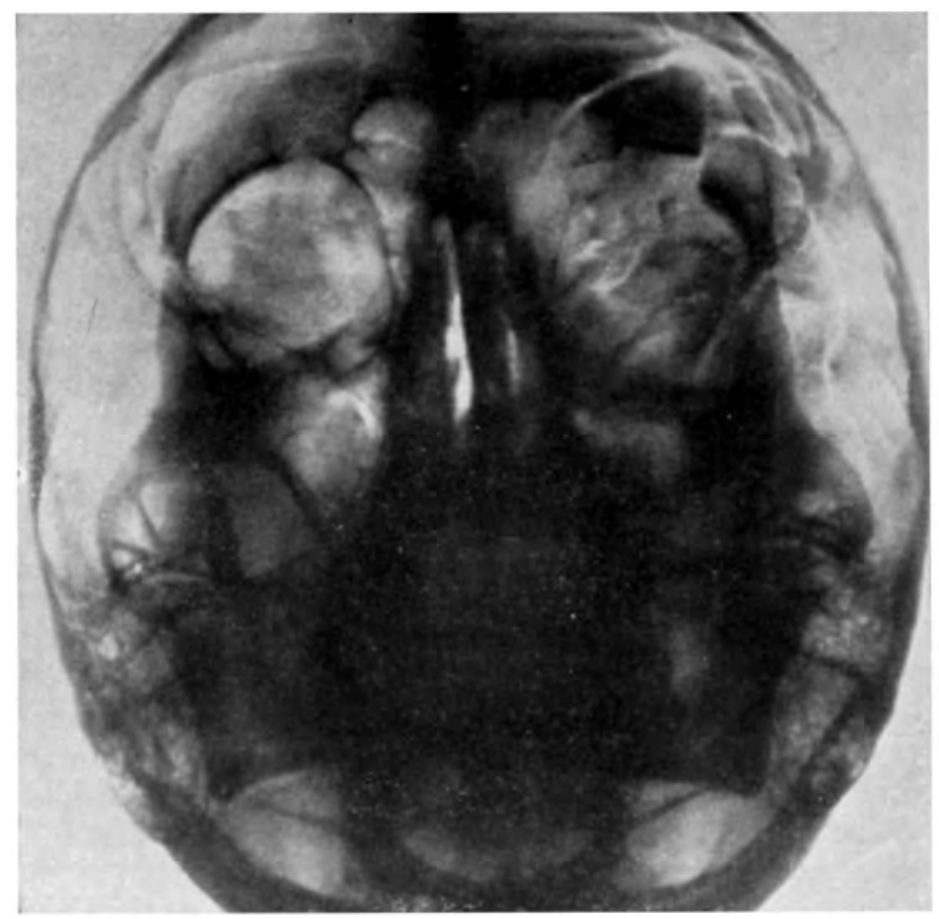

Fig. 3 - Caso 2 (H. J. K.). Radiografía A. l'., tomada bocanariz sobre la placa: fractura del techo de la órbita izquicrda; la línea de fractura llega hasta el seno frontal izquierdo; el borde externo de la órbita izquierda muestra una "frontozygomatic separation"; a causa de ello el borde inferior de la órbita izquierda está desplazado hacia abajo. 
Caso $2-$ H. J. K. Ingresó en el Hospital de Deventer ror haber sufrido un accidente de motocicleta. Estuvo sin conciencia 3 dias. En el momento en que la recobró fué transportado a Utrecht, el 24 de Mayo de 1951, para se: tratado por el cirujano plástico Dr. Honig de una fractura del maxilar sun€rior y maxilar inferior izquierdo e impresión del pómulo izquierdo. A consecuencia de una "fronto-zygomatic separation" la base de la órbita estaba desplaza hacia abajo, presentando el enfermo diplopia. Se repuso el hundimiento del cigcina y la fractura mandibular. Eil maxilar inferior fué fijado, después de lo cual nos fué enviado el enfermo por mostrar el examen también una fractura del techo de la órbita, en la que el seno frontal estaba abierto.

Exploración - Existia un exoftalmos del ojo izquierdo, que estaba además desplazado hacia abajo. 'lanto la visión como los movimientos oculares estaban intactos. El examen neurológico no mostró ninguna anomalía. I as radiografías de cráneo (fig. 3) mostraron varias lineas de fractura del frontal y del ternporal izquierdos. El techo de la órbita estaba fracturado en pedazos, alcanzando también la fractura el seno frontal. En la pared externa de la órbita existía una "fronto-zygomatic separation".

Operación -- El 3 Julio 1951, trepanación frontal izquierda. En la exploración extradural del techo de la órbita se encontró en la clura una cicatriz adherente a trozos fracturados aislados a la periórbita. En la exploración intradural se encontró en la base del lóbulo frontal una cicatriz meningocerebral. Se extirpó la cicatriz, viéndose que el cerebro hacía hernia en una abertura de la parte orbitaria del seno frontal. I a dura fué cuidadosamente cerrada. Se extirpó el trozo fracturado aislado del techo de la órbita y se cerró la abertura de la parte orbitaria del seno frontal con un trozo de músculo. El seno fué aún cubierto con una transplantación de fascia lata, después de lo cual se cerró la herida. A continuación de la operación desapareció el exoftalmos, pero el desplazamiento hacia abajo del ojo, así como la diplopia, persistieron.

El cirujano plástico, Dr. Honig, hará más tarde una elevación del fondo de la órbita para corregir la posición del ojo. Ocho meses después del trauma fué tomado un EEG̈, que mostró una actividad tetánica paroxismal, frontotemporal, predominantemente en el lado izquierdo.

En los casos de heridas penetrantes intracraneales transorbitarias, la abertura en el techo de la órbita se produce lo más frecuentemente por un objeto agudo que lo perfora a través del párpado superior (ver también McClure y Gardner). Nosotros hemos visto estas heridas sólamente en niños, a consecuencia de accidentes durante el juego en los que penetró un pequeño bastón a través del techo de la órbita. La herida en el párpado superior lo más frecuente no llama la atención por su pequeñez. El ojo no está casi nunca lesionado, dada su gran posibilidad de despla. zamiento en la órbita y la escasa velocidad de entrada del objeto penetrante. Si no se hace radiografía del techo de órbita, la herida intracraneal puede pasar desapercibida. Igualmente, si la radiografía no se toma en buena posición puede no evidenciarse en ésta la perforación. El peligro de infección es bastante grande, sobre todo si la herida se produce con objetos que han estido en contacto con la suciedad de la calle, como ocurre frecuentemente en los juegos de los niños. La punta de un bas. tón o un pizarrín puelde romperse en alguna parte y quedar introducida bajo el techo de la órbita. Los cuerpos extraños de madera ro son visibles en la radiografía. 
Sobre la base de estos hechos, creemos que está permitido recomendar, en todas las heridas penetrantes del párpado superior, las siguientes medidas: 1) Debe tomarse una radiografía de cráneo, A.P., tanto boca-nariz como nariz-frente sobre la placa. 2) Si se ha constatado una perforación del techo de la órbita debe hacerse, cuanto antes, una craniectomía frontal exploradora, para tener una buena visión de conjunto, no sólamente del techo de la órbita, sino también de la dura y de la base del lóbulo frontal. 3) También debe explorarse el contenido intraorbitario, ya que existe la posibilidad de suciedad y cuerpos extraños. No se debe dejar de hacer una exploración de la herida externa, pero sólamente esto no es suficiente. Durante la operación intracraneal, el agrandamiento de la perforación en el techo de la órbita ofrece un buen campo para examinar el contenido orbitario.

Nuestro punto de vista de operar estas heridas intracraneales penetrantes por vía transcraneal puede parecer un poco agresivo, y se nos podría decir que un tratamiento a partir de la herida del párpado superior ha dado en muchos casos buenos resultados. Nosotros creemos, sin embargo, que las dos historias clínicas siguientes of recen argumentos vivos para sostener nuestro punto de vista.

En el primer enfermo no se había explcrado primeramente nada más que la herida del párpado superior; después de єsto el enfermo fué tratodo con antibióticos, pero una meningitis recidivante, coma, exoftalmos progresivo y alteraciones ventriculográficas, exigieron una intervención transcraneal. En el otro enfermo, que había sido explorado a tiempo por via transcraneal, encontramos escondida la punta rota de un peqieño bastón de madera, introducida profundamente en la órbita; el hallazgo de este cuerpo extraño era imposible por una exploración a través del párpado superior, no pudiendo tampoco la radiografía dar la indicación de intervenir; aunque se hizo la limpieza de la herida por dos vías no pudo prevenirse la infección, lo que demuestra la gravedad de este tipo de heridas; la historia clínica de este enfermo es además muy interesante porque dá una buena idea del origen de algunas formas de exoftalmos pulsátil.

Caso 3 - W. H. ( $n^{\circ} 4966$ ), joven de 9 años. En 18 Septiembre 1948, penetró la punta de un bastón a través del párpado superior, por debajo y detrás de la ceja. El muchacho estuvo algunos minutos sin conocimiento y presentó un vómito al recobrar conciencia. Existia una herida sin importancia en el párpado superior derecho. Fué llevado a un Hospital, donde se le hizo una radiografía de cráneo (tomada frente-nariz sobre la placa) que no mostró ninguna anormalidad. Se hizo una toilet de la herida encontrándose, elı ésta, suciedad que había penetrado con el objeto contundente. El 19-9-1948, fluye líquido a través de la herida del párpado superior, que fué tomado por el cirujano por exudado de edema. El 20-9-1948, edema del párpado superior y fiebre hasta $39,55^{\circ} \mathrm{C}$; tratamiento de la herida con compresas de ácido bórico. Ei 21-9-1948, meningitis que fué tratada con penicilina intratecal e intramuscular y Cibazol por via oral. El 22-9-1948 el enfermo deviene comatoso; gran exoftalmos del ojo derecho con edema palpebral; se hizo una incisión descompresiva en el párpado superior; al día siguiente la temperatura era normal. El 6-10-1948, liquido cefalorraquideo 
normal; el exoftalmos desapareció; párpados normales; buen estado de conciencia. El 16-10-1948, recidiva de la meningitis $y$ del exoftalmos; el enfermo fu. transportado a la clínica de neurocirugía.

El 19-10-1948, ingreso en neurocirugía: exoftalmos con desviación hacia abajo del ojo derecho; gran edema del párpado superior y de la ceja. La radiografía de cráneo (fig. 4) mostró una perforación con pequeños trozos de hueso en el techo de la órbita derecha.

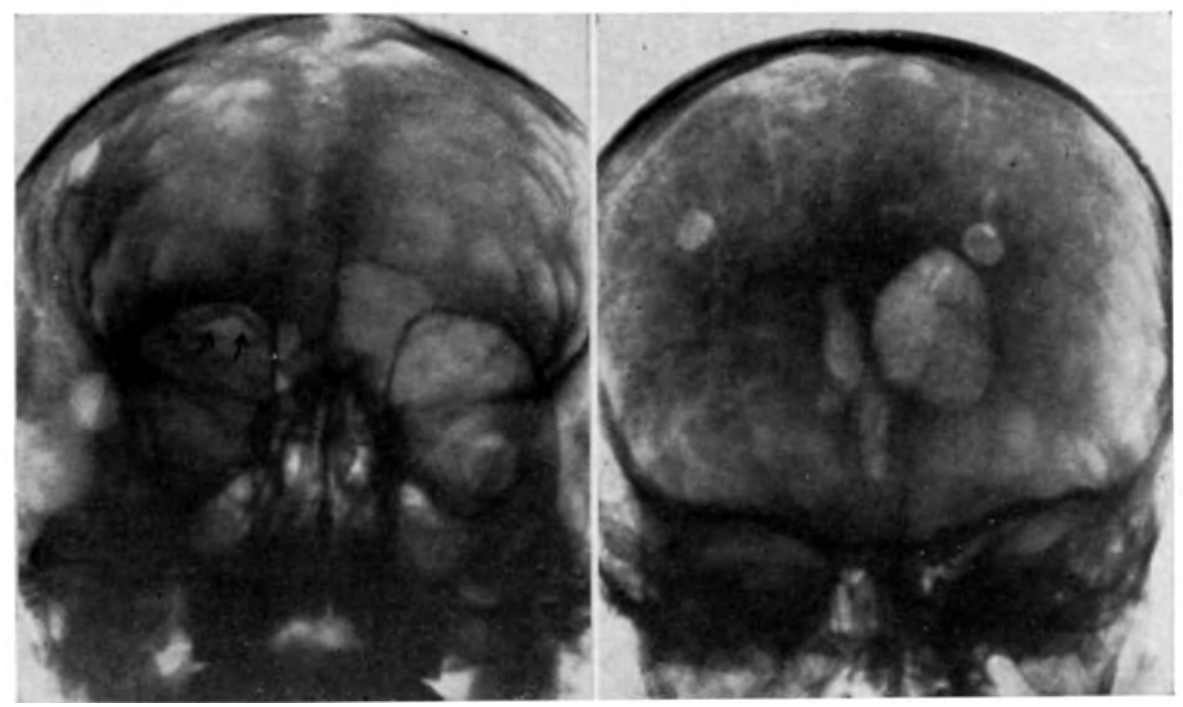

Fig. 4 - Caso 3 (W. H.). En la izquierda, herida penetrante del techo de la órbita derecha (ver la flecha). En la derecha, ventriculograma: dilatación del ventrículo lateral izquierdo; defecto lateral de replección en el cuerno frontal derecho; no existe desplazamiento del sistema ventricular.

Exploración neurológica: Dilatación de la pupila derecha, que no reacciona a la luz; paresia facial inferior izquierda; paresia del brazo izquierdo; reflejos tendinosos en los brazos, no se obtienen.

Por punción ventricular derecha, examen del líquido: 500/3 células blancas y $77 \mathrm{mg}$ de albúmina. Hxamen del líquido del ventrículo izquierdo: 488/3 células blancas y $30 \mathrm{mg}$ de proteínas. El ventriculograma muestra una dilatación del ventrículo izquierdo. El ventrículo derecho está agranclado, excepto en el territorio del cuerno anterior, donde existe una impresión lateral. Eil sistema ventricular no está desplazado enteramente. El hemisferio derecho muestra por lo tanto un proceso local, linitado al lóbulo anterior dercchs, que no va unido sin embargo a un edema cerebral generalizado. Por punción del lóbulo frontal derecho no se encontró ningún abceso.

Trepanación frontal derecha: La dura está moderadanente tensa; tampoco se encontró ningún abceso. Después de rechazar el lóbulo frontal se expuso el techo de la órbita. Existe una cicatriz cérebro-dural que se extiende tanto hacia la órbita como hacia el lóbulo frontal. Se extirpa la cicatriz, así como viejos 
coágulos del lóbulo frontal, cerrándose el defecto de dura con una plastia de fascia. Se puso penicilina local, cerrándose la herida a continuación.

El curso postoperatorio fué tranquilo. El exoftalmos desapareció gradualmente y ia posición del ojo volvió a la normalidad. El número de células del líquido se mantuvo aumentado durante un mes y medio después de la operación. En examen de control, en Diciembre 1949, no mostró el enfermo ningún dato anormal.

Caso 4 - H. H. ( $\mathrm{n}^{\circ}$ 8326), niño de 3 años. Durante el juego penetró un bastón a través del párpado superior izquierdo, hacia el interior de la órbita. El niño fué llevado en estado soporoso al Hospital, donde se suturó una pequeña herida del párpado superior. Las radiografías mostraron trozos de hueso en la fosa anterior izquierda del cráneo, señal de que el bastón había penetrado a través del techo de la órbita. El niño fué llevado el mismo dia a la clínica neuroquirúrgica, donde quedó ingresado el 22-11-1950: estaba soporoso, con el párpado superior izquierdo muy hinchado y de coioración hemática. La exploración neurológica no mostró nada importante.

Operación - Trepanación frontal izquierda. La exploraciói extradural del techo de la órbita es imposible, debido a la gran presión intradural que no se modifica por la punción ventricular. Al abrir la dura se hernió el lóbulo frontal, muy edematoso. 'Irás la amputación del polo anterior del lóbulo frontal se evidenció una extensa contusión de la parte basal de este lóbulo. Se extirpó el tejido cerebral contusionado incluidos los trozos de hueso que habían penetrado. Se agrandó la abertura en el techo de la órbita viéndose el trozo de punta del bastón que estaba escondido en la profundidad de la órbita, bajo la parte lateral del techo. El defecto de dura fué cerrado con fascia. La grasa orbitaria llenó el espacio muerto. Se administró penicilina tanto intra como extraduralmente, después de lo cual se cerró la herida. Se limpió la lesión del párpado superior, encontrándose papilla de tejido cerebral en el orificio de entrada.

El niño fué tratado con penicilina. Los primeros dias siguientes mejoró bastante el estado de conciencia. El ojo quedó cubierto por el párpado superior edematoso, pero 5 dias después se presentó una fuerte reacción meningea (15.400/3 células blancas); el líquido cefalorraquideo no contenia ningún microorganismo pero en la herida del párpado superior apareció un exudato purulento. Se aseguró un amplio desagüe del párpado superior, desapareciendo después de esto la reacción meningea durante algunos días. El 15-1-1951 el estado de conciencia era muy bueno. Existía un enoftalmos del ojo izquierdo y todos los novimientos oculares estaban intactos. El alto contenido de proteínas del líquido (120 $\mathrm{mg} \%)$ acompañado de un aumento discreto de células (75/3) mostraban que existía aún cierto grado de inflamación, aunque la presión del líquido era normal.

El 23-1-1951 comenzó el niño a presentar frecuentes vómitos, aquejando dolor de cabeza. Apareció un exoftalmos pulsátil del ojo izquierdo. Él líquido estaba amarillo ( $7 / 3$ células y $72 \mathrm{mg} \%$ de proteínas) con alta tensión. El ventriculograma (fig. 5) mostró un sistema ventricular agrandado y aire en la cisterna magna y en la cisterna pontis, pero no en la región quiasmática. El cuerno frontai izquierdo hacía hernia hacia la órbita izquierda.

El mismo dia fué explorado el quiasma por vía de una craniectomia frontal derecha. Existía una aracnoiditis quiasmática muy extensa. Se liberaron las adherencias abriéndose la lámina terminalis, aún cuando había pocas esperanzas de restablecer con esta operación ia circulación de líquido. El exoftalmos pulsátil persistió igual y el ojo sufrió una desviación hacia abajo. La protusión ocular disminuyó mucho después de una punción ventricular descompresiva. El examen del líquido ventricular mostró la existencia de una ventriculitis. El 15-2-1951 se puso el niño comatoso y presentó una hemiplejía derecha; el exoftalmos aumentó enormemente; la medida comparada de la tensión ventricular y la estimación ventricular mostraron un bloqueo del Monro izquierdo. 


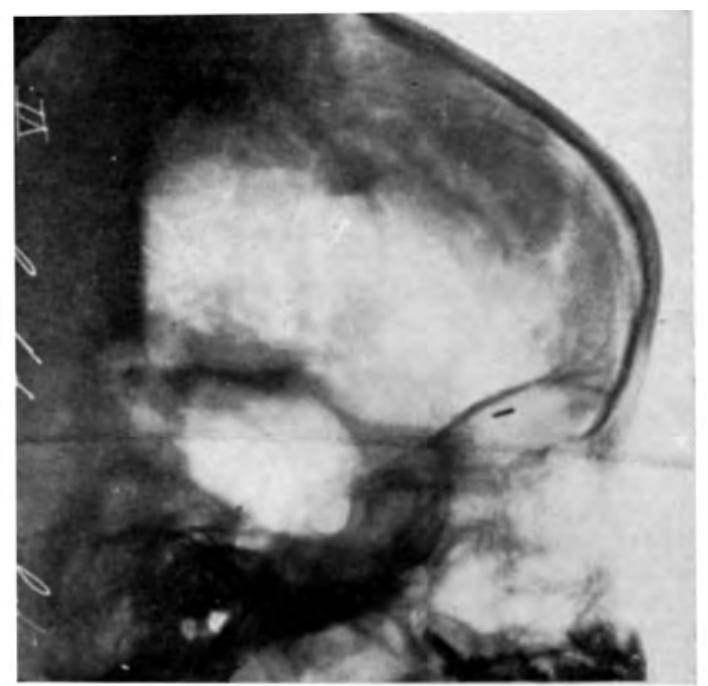

Fig. 5 - Caso 4 (H. H.). Sistema ventricular muy dilatado; el cuerno frontal izquierdo se hernia en la órbita del mismo lado.

Este ventrículo fué unido a la cisterna magna por medio de un tubo de politeno (operación de 'Torkildsen). Inmediatamente después de la operación el paciente recobró el conocimiento, la hemiplejía mejoró y el exoftalmos disminuyó. İa presión ventricular era de $270 \mathrm{~mm}$. de água, en ambos lados, existiendo, por lo tanto, una moderada elevación. Los fenómenos de meningitis basilar persistieron hasta Agosto del 1951 y, el 22 de Septiembre, pudo el niño darse de aita. Ia tensión del líquido era normal y no sólamente no existía ningún exoftamos, sino que incluso había un enoftalmos del ojo izquierdo.

Lipicrisis --- Como norma general, trás la supresión operatoria del techo de la órbita, no se presenta ningún exoftalmos si la presión intracraneal es normal. Esto prueba claramente que, en la historia clínica de este enfermo, aquél persistió sólamente durante el período de hidrocéfalo hipertensivo.

Las alteraciones descritas bajo los apartados A y B, exigen un tratamiento rápido. Es por lo tanto conveniente que estos enfermos sean transportados a centros bien equipados, donde es posible, durante el mismo tiempo operatorio, hacer un tratamiento definitivo. Debe desaconsejarse un tratamiento rápido en el lugar del accidente, pero sólamente parcial, pues ello no hace sino perder tiempo, sin disminuir el peligro de algunas complicaciones, que incluso se aumenta.

C. Comunicaciones órbitocraneales producidas de dentro hacia fuera En dos enfermos con hidrocefalia crónica producida por estenosis del acueducto se produjo una perforación del techo de la órbita, a consecuencia de un traumatismo craneal, a través de la cual protuia el lóbulo fron- 
tal, con un consiguiente exoftalmos pulsátil del ojo respectivo. Es posible que no se reconozca esta forma de exoftalmos y sea atribuido erróneamente a un aneurisma arterio-venoso si además, como sucedía en nuestros dos eitfermos, las pulsaciones disminuyen por compresión de la carótida homolateral. En estos dos enfermos existía una estenosis del acueducto que no había dado ninguna sintomatología antes del traumatismo, pero sin embargo las radiografías de cráneo mostraron las típicas alteraciones de hipertensión intracraneal crónica. El diagnóstico puede hacerse con seguridad: 1) por visualización del defecto en el techo de la órbita en la radiografía A.P. tomada tanto boca-nariz como frente-nariz sobre la placa; 2) vor la disminución del exoftalmos y las pulsaciones del ojo cuando se disminuye la presión intracraneal por medio de una punción ventricular.

El tratamiento operatorio consiste en una aproximación transcraneal al techo de la órbita, reposición o resección del prolapso cerebral intraorbitario, cierre del eventual defecto de la dura y cierre, por medio de una plastia, del defecto en el techo de la órbita, con una lámina periostiocortical. De ser posible, debe tratarse primeramente el hidrocéfalo, ya que ello da a la operación en el techo de la órbita una mejor posibilidad de éxito.

D. Deformaciones especiales de la cavidad orbitaria - Las alteraciones traumáticas del canal óptico interesan al neurocirujano sólamente en cuanto van unidas a una lesión del nervio óptico. Si se produce pérdida de visión inmediatamente después del trauma se deberá examinarlo además de verificar el estado del canal óptico ya que la perturbación es debida generalmente a una lesión de este nervio, que puede ser ocasionada por diferentes causas (hemorragias intracraneales del nervio óptico, lesión de los vasos nutritivos, fracturas del canal óptico acompañadas de deformación, herida directa del nervio óptico por trozos de hueso y, especialmente, fractura de apófisis clinoides y shock del nervio óptico). Si las alteraciones ópticas se producen más tardíamente, están generalmente originadas jor formación de callo en el canal óptico por aneurisma, por trombosis de los vasos nutricios o por aracnoiditis óptica. Si la ceguera se produce a consecuencia de una herida de los vasos nutricios del nervio óptico se puede comprobar rápidamente palidez de la papila (Walsh); en las demás heridas del nervio óptico se produce la palidez más tarde, por regla general de algunas semanas a meses y, por término medio, unos 17 días.

La frecuencia de las lesiones ópticas en los eniermos traumatizados de cráneo es, según Rowbotham, de 0,5\% y, según Turner, de 1,5\%. En la literatura oftalmológica existen bastantes differencias de opirión sobre la frecuencia de hemorragias en el canal óptico y de fiacturas del mismo como causa de pérdida traumática de visión.

En las deformaciones traumáticas evidentes del canal óptice o en el caso de una dislocación de la apófisis clinoides anterior, existe una clara indicación de intervenir. En nuestro registro no existe ningun caso aná- 
logo y su rareza se atestigua ya que, según Turner, en 33 enfermos con heridas del nervio óptico en los que se hizo radiografía del agujero óptico, sólamente 4 mostraron alteraciones radiológicas de dicha estructura. Se toma como argumento que las fracturas del canal óptico y los trozos de hueso que comprimen o penetran el nervio óptico pueden ser invisibles en la radiografía, pero, en tales casos, la indicación de intervenir es muy dudosa. Nosotros recordamos un enfermo en el que inmediatamente después del trauma se produjo una hemianopsia horizontal, hacia abajo, del ojo derecho; la radiografía mostró una iractura del cigoma derecho y una fractura lineal del techo de la órbita del mismo lado, que se aproximaba al vértice; la exploración intracraneal del nervio óptico derecho no inostró con claridad ninguna alteración; el nervio optico tenía un aspecto normal, y no existía ninguna compresión a nivel del canal.

La mayoría de las lesiones intraorbitales de los nervios oculares están producidas, en tiempo de paz, por alteraciones traumáticas de la fisura orbital superior. No insistiremos más sobre el diagnóstico. Si, en determinados casos, se puede atribuir con bastante seguridad la cftalmoplejía a una lesión de la fisura orbital superior, no deberá uno inclinarse a in. tervenir enseguida sin esperar una eventual curación espontánea de los trastornos. Cuando no se produce mejoría puede pensarse en una descompresión operatoria especialmente $\mathrm{si}$, en los trastornos de esta clase, existe una posibilidad aceptable de que la continuidad de los vasos y nervios que pasan a través de la fisura orbital superior no está interrumpida. Esto ocurrió en el siguiente enfermo:

Caso 5 -- 'Th. T. ( ${ }^{9}$ 7977), joven de 8 años. Admitido en la Clinica de Neurocirugía el 22 de Mayo de 1951. Seis semanas antes había sufrido un grave traumatismo craneal en un accidente de ascensor, después de lo cual estuvo 2 días sin conciencia. Cuando la hinchazón del párpado disminuyó se observó que el ojo derecho estaba totalmente ciego existiendo además una oftalmoplejía completa de este ojo. El cigoma derecho estaba imprimido. El niño fuć hospitalizado especialmente con vistas a la posibilidad de mejorar el trastorno oftalinológico con una intervención operatoria.

Exploración - Comportamiento psíquico normal. Buen estado de nutrición. En el lado derecho está hundida la facies malar del pómulo, asi como el borde lateral y inferior de la órbita. La visión del ojo derecho es nula. La papila derecha está fuertemente atrófica. Existe una parálisis completa del músculo elevador del párpado superior y de toda la musculatura ocular en el lado derecho. La pupila derecha es mayor que la izquierda y no reacciona a la luz ni a la convergencia. Es muy curioso que el diámetro de esta pupila, sin embargo, por causa desconocida, es variable. En el lado derecho el reflejo corneal está abolido $y$ existe una anestesia en el territorio de la primera rama del trigémino. El mủsculo frontal está paralizado. A excepción de un Rossolimi en el pié derecho, no se encontró ningún otro dato patológico.

E.camen electroencefalogrifico (Dr. Storm van Lecuwen) - Ritmo $\alpha$ irregular. Actividad $\delta$ y $\theta$ ditusas, especialmente occipital. Actividad $\theta$ de frecuencia baja, sincrónica, paroxismal, en ambos lades temporales. Por hiperventilación de corta duración se produce una parecida serie bifrontal, precedida de algunas 
ondas de dudosos picos. Examen radiológico del cráneo (fig. 6) - La órbita derecha muestra los siguientes dátos: el techo está desplazado hacia arriba a causa de un rechazamiento de ala magna hacia la línea media; la físura orbital superior está desplazada hacia la parte superomedial y es muy estrecha; la fisura orbital inferior está vertical; el agujero óptico está fuertemente rechazado hacia la línea media, pero muestra, en la posición de Reese, un diámetro rormal; el borde orbital lateral presenta una "fronto-zygomatic separation" por lo que la base de la órbita está desplazada un poco hacia abajo. Ventriculograma: discreta dilatación del sistema ventricular.

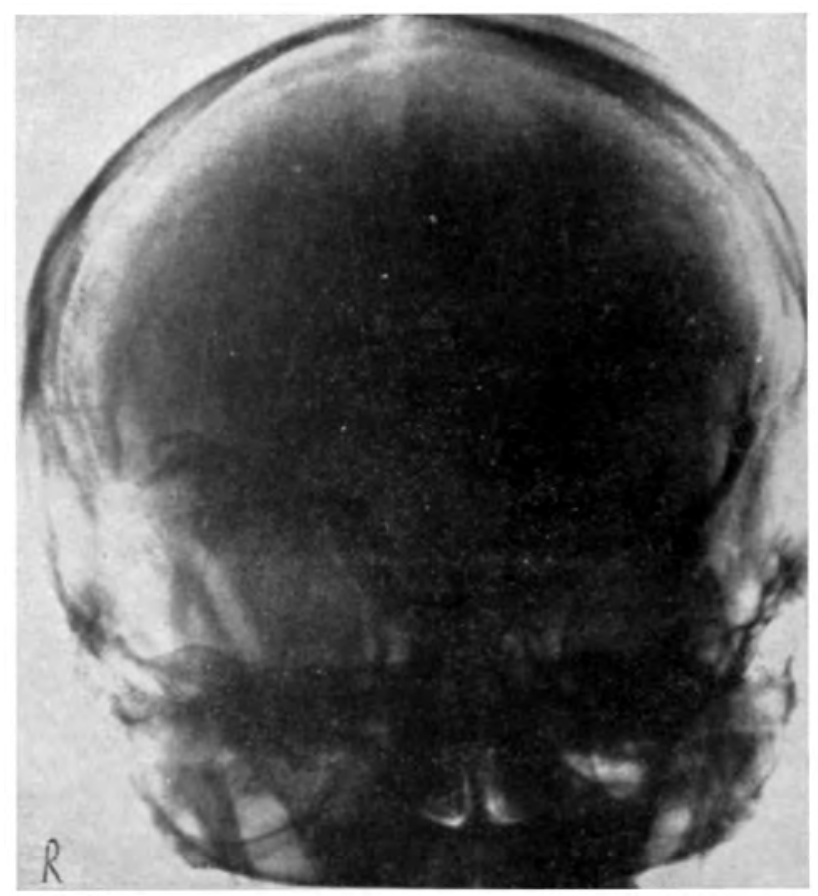

Fig. 6 -. Caso 5 ('T'h. 'T.). Impresión lateral de la órbita derecha: la fisura orbital superior está desplazada hacia la linea media y hacia abajo; el borde externo de la órbita muestra una "fronto-zygomatic separation"; la parte lateral del techo de la órbita está desplazado hacia arriba.

Puesto que la ceguera existía desde inmediatamente después del trauma, habiendo pasado ya 6 semanas, y el examen oftalmoscópico mostraba una atrofia evidente de la papila, no podía esperarse una mejoría por la intervención operatoria. Juzgamos que le reparación de la función de los múscclos oculares era posible por descompresión de la fisura orbital superior, ya que parecía que pasaban aún estímulos pupilotónicos. Puesto que los padres consideraban esta me- 
joría muy importante desde el punto de vista cosmético, se decidió la operación el 22 de Mayo de 1951.

Operación - Pequeña trepanación frontotemporal derecha. A partir de la parte anterior de la fosa media fué extirpada con la pinza gubia la mitad superior del ala magna, por lo que fueron descomprimidos los vasos y nervios que pasan a través de la fisura orbitaria. Se extirparon algunos pequeños trozos de hueso que habían perforado la dura temporal durante el trauma. La dura fué abierta. Aparte de observarse una meningitis serosa el cérebro no mostró ninguna otra anomalía. Se cerró la herida. Ėl cirujano plástico (Dr. Honig) repuso quirúrgicaniente el cigoma.

EI curso postoperativo fué tranquilo y el 23 Junio el enfermo abandonó la clínica. A fines de Junio del 1951 el músculo elevador del párpado superior comenzó a recuperarse. En Octobre eran posibles movimientos limitados del ojo derecho. En Diciembre los movimientos de los ojos eran completamente normales. En Marzo de 1952, la sensibilidad de la región de la primera rama del trigémino había recuperado completamente. Existía aún una disminución de la sensibilidad corneal derecha, pero se podía provocar el reflejo corneal, aún cuando estaba debilitado. Desde hace algún tiempo el enfermo siente de nuevo cuando penetra algún cuerpo extraño en el ojo, lo que significa cvidentemente una mayor seguridad para el mismo.

La reconstrucción plástica del borde superior de la órbita se hace tanto por consideraciones cosméticas como por las dificultades subjetivas u objetivas que algunos enfermos experimentan después del defecto. Con frecuencia puede hacerse la intervención sin ningún riesgo. Nosotros preferimos la transplantación autógena con hueso (un trozo de costilla), a la implantación con sustancias aloplásticas. Primeramente hicimos la operación sin previa encefalografía, pero tenemos una experiencia muy desfavorable en un enfermo (caso 6), por lo que estamos convencidos de la necesidad de la insuflación de una pequeña cantidad de aire.

Caso 6 - P. M. ( $\mathrm{n}^{\circ}$ 6080), de 32 años, tuvo el 24 Marzo 1949, durante una acción de guerra en Indonesia, una herida craneal penetrante por disparo de bala. La pequeña abertura de entradla de la bala estaba encima del oído dcrecho, y el crificio de salida encima del ojo del mismo lado. EI cerebro se herniaba hacia la herida frontal. El enfermo fué transportado a Samarang $y$ allí operado. Fué necesario enuclear el ojo derecho. La pared posterior y el techo de la órbita estaban frígmentados. Se extirparon los irozos aislados de hueso. Se extirpó asimismo el tejido cerebral desvitalizado, cerrándose el defecto de dura con una plastia. Al ingresar en el Hospital Militar de Utrecht no existía ningun dato neurológico de exploración, pero el enfermo estaba muy deprimido y sin iniciativa. Se decidió hacer una reconstrucción plástica del territorio orbitario lesionado. El cirujano plástico (Dr. Raadsveld) manifestó la preferencia de que la reconstrucción plástica del borde superior de la órbita fuera hecha por el neurocirujano. El paciente fué admitido en la Clínica de Neurocirugía el 5 Enero 1950.

Operación - Incisión de la ceja derecha. Se llegó a un territorio de tejido conjuntivo fibroso y duro. Se libertó la dura y se implantó una parte de la décima costilla para completar el borde superior de la órbita, fijándola al borde lateral de ia misma, que aún existía: en la parte medial la plastía no pudo fijarse al hueso frontal, porque la liberación del mismo producia el riesgo de reabrir el seno frontal. Desgraciadamente se produjo un pequeño desgarro de la dura, 
a través del cual salía bastante líquido; se suturó esta abertura, cerrándose después la herida en dos planos.

El enfermo recibió un tratamiento post-operatorio de penicilina intramuscular. Cuatro días después de la operación devino confuso, la temperatura subió hasta $39^{\circ} \mathrm{C}$ y salía mucho liquido a través de la herida. Por punción ventricular la presión del líquido era muy elevada, y éste contenia 10.400,43 células. En dias sucesivos se inyectó penicilina en el ventrículo. El 10 de Febrero el número de células del líquido ventricular había disminuido hasta $40 / 3$, pero $\epsilon$ l 14 de Febrero subió $(350 / 3)$.

Debido a que la herida exudaba continuamente líquiclo se extirpó la transplantación el 18 Febrero. Se inspeccionó nuevamente la dura pareciendo que salía líquido a través de una pequeña abertura del tamaño de una cabeza dc alfiler, observándose una gran cavidad que ocipaba toda la región frontal y entraba hasta el ventrículo. El foramen de Monro era muy grande. Se suturó cuidadosamente la dura, y la zona de sutura se cubrió con una pieza de fascia lata. Después se cerró la herida en dos planos. A partir de este monientu el enfermo devino cada ve\% más comatoso, con continua hipertermia, y murió ei 25 Febrero 1950.

Épicrisis - El fracaso de esta operación se basa en diversas causas: 1) La fistula no se podía cerrar porque debajo de la duramadre se encontraba sólamente líquido, mientras que el tejido que rodeaba a la transplantación estaba fuertemente alterado y escleroso; por ello la curación de la herida fué retardada. 2) La infección posoperatoria del ventrículo: posiblemente existia en este enfermo una resistencia disminuida contra la infección, vista también la piodermitis que había padecido. El hecho de que el ventrículo estuviera separado dei terreno operatorio sólamente por la duramadre y el origen de la fístula, eran factores favorables para la infección. 3) El trastorno de la regulación vegetativa: el equilibrio regetativo es muy lábil en los enfermos con una gran atrofía cerebral; estos enfermos están más predispuestos que otros a una hipertermia. Es conocido que estos pacientes pueden presentar violentas reacciones después de una punción ventricular, y una ventriculografia puede incluso ir seguida de la muerte. Enfermos semejantes reaccionan en general muy desfavorablemente a las alteraciones del equilibrio de la presión intracraneal y esto explica igualmente porqué la fístula de líquido fué seguida de un curso tan extraordinariamente desfavorable en nuestro enfermo.

En virtud de esta experiencia, nosotros creemos conveniente hacer una encefalografía con una pequeña cantidad de aire antes de cumenzar una reconstrucción plástica del borde superior de la órbita. En una atrofia grande del lóbulo frontal con acumulación de líquido àebajo de la duramadre, en la región del terreno operatorio, la intervención ofrece grandes riesgos.

\section{BIBLIOGRAFIA}

Calvert, C. A. - Orbito-facio-cranial gunshot wounds. Brit, J. Surg., War Surg. Suppl., 1:119-141.

McClure, C. C. y Gardner, W. J. - 'Transorbital intracranial stab wounds. Cleveland Clin. Quart., 16:118-125, 1949.

Peiper, H. - Die Behandlung der Schussverletzungen des Gehiins, insbesondere der orbitalen Basisschüsse. Stuttgart, Ferd. Enke, 1944. 
Stewart, O. W. y Bottereil, E. H. -- Cranio-facial-orbital wounds involving paranasal sinuses: primary definite surgical treatment. Brit. J. Surg., War Surg. Suppl., 1:112-118.

'Tönnis, W., Seifert, E. y Riechert, T. - Kopf verletzungen. Mürchen, Lehmanns Verlag, 1943.

'Turner, A. J. W. -- Indirect injuries of the optic nerve. Brain, 66:140-151, 1943.

Walsh, F. B. - Clinieal Neuro-Ophthalmology. Williams \& Wilkins Co, Baltimore, 1947.

Webster, J. E. y Gurdjian, E. S. - Penetrating cranial wounds: a summary of methods used in management. Collective review. Surg., Gynec. a. Obst., $82: 353-380$, 1946.

Webster, J. E., Schneider, R. C. y Lofstrom, J. E. - Observations upon the management of orbito-cranial wounds. J. Neurosurg., 3:329-336, 1946.

Willem Barentzstraat, 2 - Utrecht, Nederland (Holanda). 